\title{
Competition between pure dephasing and photon losses in the dynamics of a dot-cavity system
}

\author{
A. Vagov,,${ }^{1, *}$ M. Glässl, ${ }^{1}$ M. D. Croitoru, ${ }^{1,2}$ V. M. Axt, ${ }^{1}$ and T. Kuhn ${ }^{3}$ \\ ${ }^{1}$ Institut für Theoretische Physik III, Universität Bayreuth, 95440 Bayreuth, Germany \\ ${ }^{2}$ Departement Fysica, Universiteit Antwerpen, Groenenborgerlaan 171, B-2020 Antwerpen, Belgium \\ ${ }^{3}$ Institut für Festkörpertheorie, Westfälische Wilhelms-Universität Münster, 48149 Münster, Germany
}

(Received 8 October 2013; revised manuscript received 4 July 2014; published 27 August 2014)

\begin{abstract}
We demonstrate that in quantum-dot cavity systems, the interplay between acoustic phonons and photon losses introduces novel features and characteristic dependencies in the system dynamics. In particular, the combined action of both dephasing mechanisms strongly affects the transition from the weak- to the strong-coupling regime as well as the shape of the spectral triplet that represents the quantum-dot occupation in Fourier space. The width of the central peak in the triplet is expected to decrease with rising temperature, while the widths and heights of the side peaks depend nonmonotonically on the dot-cavity coupling.
\end{abstract}

DOI: 10.1103/PhysRevB.90.075309

PACS number(s): 78.67.Hc, 03.65.Yz, 42.50.Pq, 63.20.kk

\section{INTRODUCTION}

High-quality quantum-dot (QD) cavity systems allow the realization of various technologically important devices such as sources for single [1] or entangled photons [2,3] that are relevant for applications in quantum information processing [4] as well as for tests of fundamental aspects of quantum mechanics [5]. As state-of-the-art cavities [6] have now reached a quality where the unavoidable phonon-related loss channels can compete with cavity losses, studies of the phonon influence on these systems has become a focus of topical research. It has been shown that the QD acoustic phonon coupling is responsible for an unexpectedly strong QD-cavity coupling for cavities that are detuned from the QD resonance [7-9], which manifests itself in a spectral broadening of the Mollow sidebands through off-resonant cavity emission $[10,11]$. Further, it was demonstrated that phonons strongly influence the photon statistics [12-15], can cause LO phonon-assisted Rabi oscillations [16], and lead to an enhanced coherent scattering as well as to an offresonant sideband narrowing $[10,17]$. Recently, we performed a numerically complete path-integral analysis dealing with the limiting case of negligible cavity losses and demonstrated that in this case, due to the phonon coupling, an increase of the light-matter coupling can reduce the visibility of Jaynes-Cummings revivals and can lead to a broadening and lowering of spectral sidebands [14].

The large mismatch that is typically found between phonon and dot transition energies suppresses the phonon-induced energy relaxation. Therefore, the dominant influence of phonons on the dynamics of QDs typically stems from pure dephasing processes which result from couplings of acoustic phonons to the electronic occupations and do not involve electronic transitions. Consequently, in the dynamical equations pure dephasing enters only via the equations for the off-diagonal elements of the electronic density matrix [18]. Apart from contributing to the loss of coherence, pure dephasing interactions lead to dressing phenomena where the bare electronic states pick up admixtures of phonons and thus form polaronic states [19]. This dressing is reflected, e.g., in

\footnotetext{
*alexei.vagov@uni-bayreuth.de
}

polaronic shifts of transition energies or in a renormalization of the Rabi frequency [20-23]. The latter implies that pure dephasing due to phonons can be used to modify to some extent the interaction of a quantum dot with a cavity photon. Recently, it has been suggested to use these properties for active control purposes ranging from an performance optimization of broad emitter-based single-photon sources to the development of a versatile device that can be operated either as a single-photon source or as a laser [24].

In this paper, we demonstrate that the competition of the phonon-induced decoherence with cavity losses brings in qualitatively new aspects to the system dynamics compared to the idealized cases when one of the two mechanisms is absent. In particular, the interplay between phonon-induced pure dephasing and phonon-induced renormalizations of the light-matter coupling with cavity and radiative losses strongly affects the transition from the weak- to the strong-coupling regime. The crossover from weak to strong coupling can be monitored most easily by analyzing the electronic density matrix in Fourier space. Assuming a mean photon number below 1, which is realized in many typical QD-cavity experiments, the resulting spectra comprise three lines in the strong-coupling regime and only one line in the weak-coupling regime. The side peaks appearing in the spectral triplet for strong coupling describe vacuum Rabi oscillations, while the center line represents incoherent relaxation processes that proceed without oscillations. Thus, the widths of the side peaks reflect the damping of the Rabi oscillations, while the width of the central peak is associated with the rate at which the incoherent relaxations take place.

In the present paper, we investigate how the strength of the dot-cavity coupling and the temperature affect the widths of these peaks as well as the position of the bifurcation point which separates the strong- from the weak-coupling regime. Most striking is the finding that the width of the central peak acquires a temperature dependence which would not be there in both limiting cases where either pure dephasing or cavity losses and radiative decay are absent. Interestingly, the width decreases with rising temperature. Our analysis combines a microscopic calculation of phononinduced pure dephasing effects with a phenomenological treatment of other loss channels, providing an easy link to the standard analysis of the crossover between the weak- and 
strong-coupling regimes, which in the literature is usually discussed without accounting for pure dephasing (see, e.g., Ref. [25]).

\section{MODEL AND RATE EQUATIONS}

The assumption of mean cavity-photon numbers below 1 allows us to restrict the relevant state space to the following three active states: $|0\rangle=|G, n=0\rangle,|1\rangle=|X, n=0\rangle$, and $|2\rangle=|G, n=1\rangle$, where $|G\rangle$ denotes the ground state of the dot (empty dot) and $|X\rangle$ is an excited state with a single exciton. The index $n$ refers to the number of photons in the cavity mode. Other dot states are coupled off-resonantly and are therefore neglected. The Jaynes-Cummings model [25] describes the coupling between states $|1\rangle$ and $|2\rangle$ due to the dipole interaction between the cavity mode and the QD. The third state, $|0\rangle$, is connected to $|1\rangle$ via the radiative decay process, which returns the excited state $|X\rangle$ to the ground state. In addition, $|0\rangle$ is connected to $|2\rangle$ due to cavity losses which remove the photon from state $|2\rangle$. Finally, pure dephasing interactions couple the electronic occupations of the QD to phonon degrees of freedom. Most important, such couplings do not introduce couplings between the electronic states [18,26,27]. Nevertheless, transitions induced by the cavity mode are accompanied by a large production of acoustic phonons, which causes pure dephasing in the electronic subsystem.

The simplest way to account for cavity losses, radiative decay, and the dot coupling to acoustic phonons is provided by the standard Lindblad formalism [28,29]. Here, we shall concentrate on the case where the dot transition is in resonance with the cavity mode. The resonant excitation is most favorable for observing vacuum Rabi oscillations, the presence of which indicates the transition to the strong-coupling regime. For this case, we obtain the following explicit equations for the densitymatrix elements that are defined as expectation values $\rho_{\ell j}=$ $\left\langle\hat{\rho}_{\ell j}\right\rangle$ of the operator $\hat{\rho}_{\ell j}=|\ell\rangle\langle j|$ :

$$
\frac{d}{d t}\left(\begin{array}{c}
\rho_{11} \\
\rho_{22} \\
V
\end{array}\right)=\underbrace{\left(\begin{array}{ccc}
-r & 0 & i g \\
0 & -\kappa & -i g \\
2 i \tilde{g} & -2 i \tilde{g} & -(\kappa+r) / 2-\gamma
\end{array}\right)}_{=: M}\left(\begin{array}{c}
\rho_{11} \\
\rho_{22} \\
V
\end{array}\right),
$$

where $V=\rho_{12}-\rho_{21}, g$ denotes the bare QD-cavity coupling strength, $\kappa$ accounts for cavity losses and can be expressed in terms of the quality factor $Q$ and the dot transition frequency $\omega_{0}$ as $\kappa=\omega_{0} / Q, r$ is the radiative decay rate, and $\gamma$ is introduced to account for the phonon-induced pure dephasing [24,29]. The pure dephasing origin of $\gamma$ is reflected in the fact that, unlike $\kappa$ and $r$, it does not enter the equations for the occupations $\rho_{11}$ and $\rho_{22}$. The dynamics of the relevant density-matrix elements involving the ground state $|0\rangle$ does not couple back to the above variables and thus does not need to be considered here. The Lindblad formalism, however, does not account for the frequency renormalizations that are known to result from the dressing of the electronic states with acoustic phonons due to the pure dephasing coupling $[8,14,17,20,21]$. As a phenomenological way to describe these renormalizations, we have introduced the modified coupling constant $\tilde{g}$ in our model. In accordance with the microscopic treatment, the phonon-induced modifications reflected by $\tilde{g}$ are applied only in the equation for the off-diagonal density-matrix elements.

Before discussing how this phenomenological relaxation model is related to more microscopic approaches, it is useful to briefly summarize some of its basic features. An overview of the behavior of the dissipative system described by Eq. (1) is most easily obtained by looking at the eigenvalues $\lambda$ of the matrix $M$ in Eq. (1) since the mode vectors $\mathbf{v}_{\lambda}(t):=$ $\mathbf{e}_{\lambda} \exp (\lambda t)$, with $\mathbf{e}_{\lambda}$ being the eigenvectors of $M$, span the solution space of Eq. (1). While analytic expressions for these eigenvalues are available, in the general case, when all rates in Eq. (1) are nonzero, they are quite lengthy and not instructive. This is different in two limiting cases. First, when pure dephasing is negligible, i.e., in the limit $\gamma=0$ and $\tilde{g}=g$, we recover the well-known result [30,31]:

$$
\lambda_{0}=-\frac{\kappa+r}{2}, \quad \lambda_{ \pm}=\lambda_{0} \pm \frac{1}{2} \sqrt{(\kappa-r)^{2}-16 g^{2}} .
$$

$\lambda_{0}$ is real, corresponding to an eigenmode that decays with the average rate of cavity losses and radiative decay. This mode never shows oscillations and thus contributes in the strong-coupling regime to the central peak in the spectral representation of the density matrix. The eigenvalues $\lambda_{ \pm}$ become complex for $4 g>|\kappa-r|$; that is, for large enough $g$, these modes oscillate. Looking at the real parts of $\lambda_{ \pm}$ reveals that for $4 g>|\kappa-r|$ these modes exhibit the same $g$-independent damping as the $\lambda_{0}$ mode. Thus, the $\lambda_{ \pm}$modes correspond to the side peaks in the spectral representation, which describe vacuum Rabi oscillations. Furthermore, Eq. (2) predicts that the bifurcation point $g=|\kappa-r| / 4$ which separates the weak-coupling regime from the strong-coupling regime can, even for finite $\kappa$, be shifted to arbitrarily small values of $g$ when $\kappa$ and $r$ are similar as it is the absolute value of the difference $\kappa-r$ which matters. Thus, the influences of different relaxation channels can compensate their effects on the position of the bifurcation point. In typical QD cavity systems, $\kappa$ dominates by far, and therefore, the competition between $\kappa$ and $r$ will lead only to marginal features under commonly encountered conditions. However, we shall see that a similar compensation takes place due to the competition of the damping $\gamma$ induced by pure dephasing and the relaxation channels described by $\kappa$ and $r$. Finally, if the coupling is less than $g=|\kappa-r| / 4$, the eigenvalues $\lambda_{ \pm}$are also real, and all modes are monotonically decaying. However, unlike $\lambda_{0}$, the real values of $\lambda_{ \pm}$below the bifurcation point depend on $g$.

The second simple limiting case is obtained by setting $\kappa=$ $r=0$ and keeping $\gamma$ finite. This situation is rarely discussed because, typically, $\kappa$ cannot be neglected when compared with phonon-induced pure dephasing. Of course, further progress in increasing the quality factors of cavities might make this regime accessible. Here, however, we are interested in this limit mainly for two reasons: first, it turns out to be instructive for later comparisons, and second, it will allow us to match the results of microscopic calculations of pure dephasing to the phenomenologically introduced values of $\gamma$ and $\tilde{g}$. Indeed, for $\kappa=r=0$ the eigenvalues of the matrix $M$ are given by

$$
\lambda_{0}=0, \quad \lambda_{ \pm}=-\frac{\gamma}{2} \pm \frac{1}{2} \sqrt{\gamma^{2}-16 g \tilde{g}},
$$




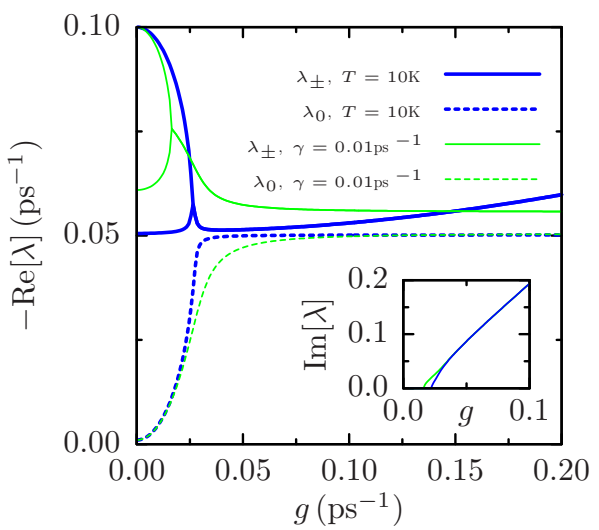

FIG. 1. (Color online) Real parts of the eigenvalues of the matrix $M$ in Eq. (1) as a function of the bare light-matter coupling $g$ calculated with $\kappa=0.1 \mathrm{ps}^{-1}$ and $r=0.001 \mathrm{ps}^{-1}$ assuming a constant pure dephasing rate $\gamma=0.01 \mathrm{ps}^{-1}$ and $\tilde{g}=g$ (thin green lines) and using $\gamma$ and $\tilde{g}$ obtained from path-integral calculations for the microscopic model of a spherical GaAs dot with radius $3 \mathrm{~nm}$ at $T=10 \mathrm{~K}$ (thick blue lines). The inset shows the imaginary part of $\lambda_{+}\left(\operatorname{Im}\left[\lambda_{0}\right]=0, \operatorname{Im}\left[\lambda_{-}\right]=-\operatorname{Im}\left[\lambda_{+}\right]\right)$.

revealing that pure dephasing alone does not damp the $\lambda_{0}$ mode, only the $\lambda_{ \pm}$modes. For any finite and constant $\gamma$, we obtain a well-defined bifurcation point at $16 g \tilde{g}=\gamma^{2}$, and for larger $g \tilde{g}$, the damping of the oscillating modes becomes independent of the light-matter coupling and is given by $\gamma / 2$.

In the intermediate case, when $\kappa, r$, and $\gamma$ are nonzero, the situation changes qualitatively, even when the frequency renormalization is neglected $(\tilde{g}=g)$. This is illustrated in Fig. 1, which displays the real parts of the eigenvalues of $M$ as functions of $g$ (thin green lines). In accordance with typical experimental values [32,33] we have set $\kappa=0.1 \mathrm{ps}^{-1}$, $r=0.001 \mathrm{ps}^{-1}$. Furthermore, we have used $\gamma=0.01 \mathrm{ps}^{-1}$. In the calculations, all rates $(\gamma, \kappa, r)$ are taken to be independent of $g$. In contrast to the limits in Eqs. (2) and (3), Fig. 1 demonstrates that, in general, the real part of all eigenvalues, which determines the damping of the modes, also changes with $g$ in the strong-coupling regime. In particular above the bifurcation point (where $\operatorname{Re}\left[\lambda_{+}\right]=\operatorname{Re}\left[\lambda_{-}\right]$), $\left|\operatorname{Re}\left[\lambda_{ \pm}\right]\right|$ decreases with rising $g$, while in both limits $\gamma=0$ and $\kappa=r=0$ a $g$-independent damping is expected for all modes when the rates $\gamma, \kappa$, and $r$ are constants.

The competition between the different decay channels also strongly affects the position of the bifurcation point. Here, it is essentially only the competition between $\kappa$ and $\gamma$, as $r$ is too small to have a noticeable influence. For the chosen parameters, the bifurcation point is found at $g_{b} \simeq 0.017 \mathrm{ps}^{-1}$, which should be compared with the bifurcation point defined by Eq. (2) that is given by $\bar{g}_{b}=|\kappa-r| / 4 \simeq 0.025 \mathrm{ps}^{-1}$; that is, adding a constant pure dephasing rate to the cavity loss shifts the bifurcation point to lower values, similar to the effect of increasing $r$ in Eq. (2) from zero to a finite value that is still well below $\kappa$.

Finally, Fig. 1 reveals that for finite values of $\gamma$ the situation is, in general, also qualitatively different from the $\gamma=0$ case with respect to the degree of degeneracy of the bifurcation point. While, according to Eq. (2) for $\gamma=0$, the eigenvalues of all three modes coincide at the bifurcation point, for finite $\gamma$, only the eigenvalues of two modes are degenerate. The two degenerate modes are those that, above the bifurcation point, correspond to oscillating modes with mutually complex conjugate eigenvalues. Also, below the bifurcation point, in Fig. 1 we have denoted the eigenvalues of those modes that continuously connect to the oscillating modes by $\lambda_{ \pm}$, while the mode that is nonoscillating for all $g$ is labeled by $\lambda_{0}$. Thus, increasing the threefold degeneracy of the bifurcation point by a finite value of $\gamma$ also allows us to unambiguously trace the oscillating modes and the nonoscillating mode separately in the region below the bifurcation point. For $\gamma=0$ such a distinction is arbitrary as all modes are continuously connected to the bifurcation point. In fact, the labeling of the modes in Eq. (2), which seems to be most natural for the case $\gamma=0$, interchanges the $\lambda_{+}$and $\lambda_{0}$ modes in the weak-coupling regime compared with what is obtained by our above assignment in the limit $\gamma \rightarrow 0^{+}$. This can be seen most clearly by looking at the results for $g=\tilde{g}=0$. In this more or less trivial case the matrix $M$ is already diagonal, and the eigenvalues are given by $-r,-\kappa$, and $-(\kappa+r) / 2-\gamma$, respectively. Physically, this corresponds to the case where the cavity is decoupled from the dot, and therefore, the dynamics reflects only the incoherent decay of the dot occupation $\rho_{11}$ described by the radiative decay rate $r$ and the disappearance of cavity photons reflected by a decrease of $\rho_{22}$ with the rate $\kappa$. The third eigenvalue $-(\kappa+r) / 2-\gamma$ describes the decay of coherences between states $|1\rangle$ and $|2\rangle$. As seen from Fig. 1, the mode that is nonoscillating for all values of $g$ is always the mode with the weakest damping for finite $\gamma$. Thus, in the limit $g \rightarrow 0^{+}$ the eigenvalue $\lambda_{0}$ of this mode approaches the value closest to zero, which for our parameters is $-r$. However, according to the assignment in Eq. (2) for $\gamma=0$ it is $\lambda_{+}$which approaches $-r$ for $g \rightarrow 0$, while $\lambda_{0}$ is given by the constant $-(\kappa+r) / 2$. We note in passing that in the limit $\kappa=r=0$ our assignment of $\lambda_{0}$ below the bifurcation point with the continuation of the nonoscillating mode coincides with the notation in Eq. (3).

\section{PHONON-INDUCED PURE DEPHASING RATES AND RABI-FREQUENCY RENORMALIZATIONS}

While cavity losses and radiative decay are usually well described by the Lindblad relaxation model [8,29-31], a description based on Markovian rates might seem, at first glance, to be questionable for phonon-induced pure dephasing processes. Indeed, pure dephasing is known to be responsible for non-Markovian effects [8,12,17,20,34], as can be seen, e.g., from the fact that the phonon-induced polarization decay after a short pulse excitation of a QD is only partial and nonexponential [18]. In this paper, however, we study the case of a cavity with at most a single photon, which is formally equivalent to a two-level QD without cavity, subject to laser driving with a constant amplitude [14]. Here, it turns out that the stationary state reached at long times is characterized by nonvanishing off-diagonal elements of $\rho$ reflecting a relaxation into a thermal distribution of the dressed states [35]. However, for resonant driving, the nonvanishing part of $\rho_{12}$ equals $\operatorname{Re}\left[\rho_{12}\right]$, which, in this case, is dynamically decoupled from the three dynamical variables $\left(\rho_{11}, \rho_{22}\right.$, and $\left.V\right)$ appearing in Eq. (1). The phonon-induced pure dephasing has recently 
been studied for QD cavity systems [14] within a numerically complete path-integral approach [36] in the limit of vanishing cavity and radiative losses. Considering a situation with at most one photon, it turns out that for a cavity in resonance with the dot transition, the path-integral results for the dynamical variables $\rho_{11}, \rho_{22}$, and $V$ agree perfectly with those obtained from Eq. (1), provided $\gamma$ and $\tilde{g}$ are suitably chosen.

Although the comparison with the path-integral results indicates that for our quantities of interest, the Lindblad approach is also valid for pure dephasing, it should be noted that in the microscopic theory both the damping and the frequency renormalization of the Rabi oscillations depend nontrivially on the bare dot-cavity coupling $g$ as well as on the temperature $T$. This dependence has been neglected in the calculations presented in Fig. 1, where constant rates have been used. We will now show that accounting for these dependencies is crucial.

Assuming that the interplay with other loss mechanisms has only small effects on the values of $\gamma$ and $\tilde{g}$, the latter can be extracted from our path-integral calculations performed for $\kappa=r=0$. To this end, we consider the path-integral solution for a system initially prepared in state $|2\rangle$, where the exciton occupation resulting from the simulation can be perfectly described by the formula [14]

$$
\rho_{11}=\frac{1}{2}\left[1-e^{-\Gamma t} \cos (\omega t)\right],
$$

where the parameters $\Gamma$ and $\omega$ are obtained from fits to the numerical path-integral data. Both parameters depend on the bare cavity-dot coupling $g$, the temperature $T$, and the dot parameters that enter the carrier-phonon coupling. Further, it was found from the path-integral analysis that, for $\kappa=r=$ 0 , the system exhibits oscillations for all $g$. This finding of oscillatory solutions in the microscopic theory for arbitrary small $g$ implies that the $g$-dependent renormalizations of $\Gamma$ and $\omega$ prevent the system from having a crossover to the weakcoupling limit; that is, unlike the theory with a $g$-independent pure dephasing rate [cf. Eq. (3)], there is no bifurcation point in a model where phonon-induced pure dephasing is the only loss channel.

In order to relate the values of $\Gamma$ and $\omega$ to the parameters $\gamma$ and $\tilde{g}$ appearing in Eq. (1) we shall choose the latter in such a way that the corresponding solution of Eq. (1) for $\kappa=r=0$ coincides with Eq. (4). We recall that oscillating solutions of Eq. (1) are found only above the bifurcation point, where, according to Eq. (3), the solution oscillates for $\kappa=r=0$ with the frequency $\omega=\frac{1}{2} \sqrt{16 g \tilde{g}-\gamma^{2}}$ and exhibits a damping of $\Gamma=\frac{\gamma}{2}$. Thus, in the case $\kappa=r=0$ Eq. (1) reproduces Eq. (4) provided we identify

$$
\begin{gathered}
\gamma(g, T)=2 \Gamma(g, T), \\
\tilde{g}(g, T)=\left[\Gamma(g, T)^{2}+\omega(g, T)^{2}\right] /(4 g) .
\end{gathered}
$$

We have determined $\gamma(g, T)$ and $\tilde{g}(g, T)$ from simulations of the dynamics for $\kappa=r=0$ that can be performed within the path-integral formalism [36] without further approximation to the model. We have used material parameters for a spherical GaAs dot with a radius of $3 \mathrm{~nm}$ coupled to acoustic phonons via the deformation potential [37]. Although the phonon coupling in GaAs-type materials is relatively weak, the corresponding
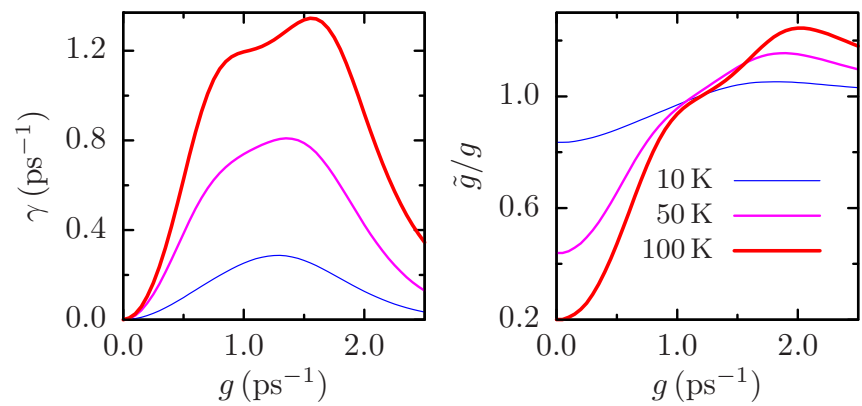

FIG. 2. (Color online) Pure dephasing damping rate $\gamma(g)$ and renormalized light-matter coupling $\tilde{g}(g) / g$ obtained as a function of the bare light-matter coupling $g$ by fitting path-integral calculations for the microscopic model at three temperatures using Eqs. (4)-(6).

effects are quite pronounced. The resulting values of $\gamma(g, T)$ and $\tilde{g}(g, T) / g$ are plotted in Fig. 2 as functions of $g \cdot \gamma(g, T)$ increases with rising $T$ and is a nonmonotonic function of $g$, reaching zero in both the small and the large coupling limits. The latter reflects the resonant nature of the dot-phonon coupling that is known from earlier works [38,39]. Also the renormalized light-matter coupling $\tilde{g} / g$ notably depends on $g$ and the temperature, where the largest deviations from the unrenormalized value of 1 are found for small $g$. Even in the present case of relatively weak dot-phonon coupling, $\tilde{g} / g$ is already, at $T=10 \mathrm{~K}$, reduced to $\sim 0.8$ for small $g$, and the reduction significantly increases with rising temperature.

As the light-matter coupling also enters the radiative recombination rate, $r$ should exhibit a corresponding $g$ dependence, which we will, however, neglect due to the overall smallness of $r$. Cavity losses, on the other hand, are mainly properties of the cavity, and hence, no significant influence of $g$ is expected. Therefore, we shall in the following use the model in Eq. (1) with $\gamma(g, T)$ and $\tilde{g}(g, T)$ determined by our microscopic path-integral approach and keep $\kappa=0.1 \mathrm{ps}^{-1}$ and $r=0.001 \mathrm{ps}^{-1}$ fixed.

\section{COMBINED IMPACT OF DIFFERENT LOSS CHANNELS}

Accounting for the $g$ and $T$ dependences of $\gamma$ and $\tilde{g}$ in Eq. (1) has significant consequences on the corresponding dependences of the eigenvalues $\lambda_{ \pm, 0}$. This can be demonstrated by comparing the results obtained with $\tilde{g}=g$ and a constant $\gamma$ (see the thin green lines in Fig. 1) with calculations accounting for the $g$ dependence of $\gamma(g, T)$ and $\tilde{g}(g, T)$ as extracted from path-integral simulations for $T=10 \mathrm{~K}$ (thick blue lines in Fig. 1). The bifurcation point is now slightly above the value expected from Eq. (2) (i.e., vanishing phonon influence) and not below, as expected for a constant $\gamma$. The same trend is seen when looking at Fig. 3, which displays the eigenvalues $\lambda_{ \pm}$for different temperatures accounting for the $g$ dependences of $\gamma$ and $\tilde{g}$. Obviously, the bifurcation point shifts to higher $g$ values with rising temperatures, which has been found previously [8] and confirms the intuitive expectation that at higher temperatures it is harder to reach the strong-coupling regime.

It is interesting to note that when only the $g$ dependence of $\gamma$ is taken into account while the renormalization of the 


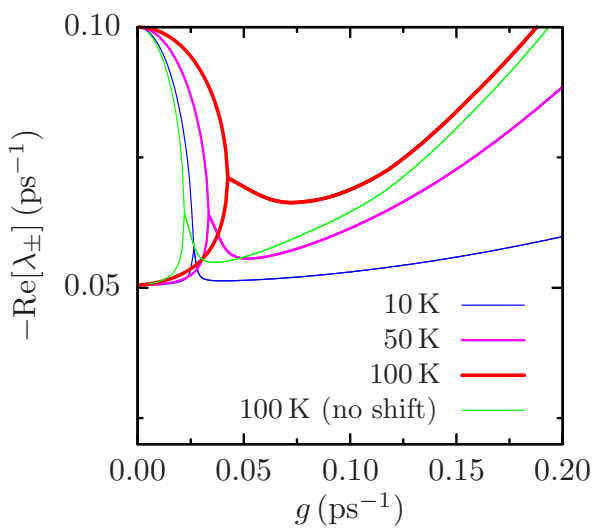

FIG. 3. (Color online) Real parts of the eigenvalues $\lambda_{ \pm}$of the matrix $M$ in Eq. (1) calculated at selected temperatures with parameters $\kappa=0.1 \mathrm{ps}^{-1}$ and $r=0.001 \mathrm{ps}^{-1}$ and using values for $\gamma(g, T)$ and $\tilde{g}(g, T)$ obtained from the microscopic path-integral calculations. The thin green line labeled "no shift" represents a calculation accounting for $\gamma(g, T)$ at $T=100 \mathrm{~K}$ but without the renormalization of the light-matter coupling, i.e., $\tilde{g}=g$.

light-matter coupling is neglected (i.e., $\tilde{g}=g$ ), the opposite trend is found (see the thin green line in Fig. 3). The latter result is due to the fact that with rising $T$, the phonon-induced damping $\gamma$ increases (see Fig. 2) and becomes comparable to $\kappa$. Thus, the situation is analogous to the competition between $\kappa$ and $r$ in the phonon-free case $(\gamma=0, \tilde{g}=g)$, where we have found from Eq. (2) that the bifurcation point can be lowered to arbitrarily small values when $r$ approaches $\kappa$. Here, we obtain a similar reduction of the bifurcation point for a relatively small $r$ when $\gamma$ approaches $\kappa$. However, this reduction due to the competition between the damping channels associated with $\kappa$ and $\gamma$ is not visible in the full calculation where, in addition, the renormalization of the light-matter interaction $\tilde{g}(g, T)$ is accounted for. As can be seen from Fig. 2 for a bare coupling $g$ well below $1 \mathrm{ps}^{-1}$, the light-matter coupling is reduced due to the phonon renormalization. Thus, compared with the phononfree case, a larger $g$ is needed to obtain a renormalized coupling $\tilde{g}$ that is sufficient to reach the strong-coupling regime. Thus, the respective influences of the pure dephasing rate $\gamma(g, T)$ and the renormalization of the light-matter coupling $\tilde{g}(g, T)$ shift the position of the bifurcation point in opposite directions. Obviously, the effect due to the reduction of the light-matter coupling is larger, which eventually leads to a shift of the bifurcation point towards larger light-matter couplings with rising temperature.

Another striking feature seen in both Figs. 1 and 3 is that when all $g$-dependent phonon-induced renormalizations are included, we now find that the damping of the oscillatory modes $\lambda_{ \pm}$increases with increasing $g$ above the bifurcation point, which is the opposite of the trend seen in calculations with a $g$-independent $\gamma$ (thin green lines in Fig. 1).

Finally, we note that the $g$ range displayed in Figs. 1 and 3 represents roughly the range of values for $g$ that has been realized in typical state-of-the-art cavities [32,33]. It is worthwhile to mention that when $g$ is further increased, $\operatorname{Re}\left[\lambda_{ \pm}\right]$ eventually decreases again, mainly due to the nonmonotonic

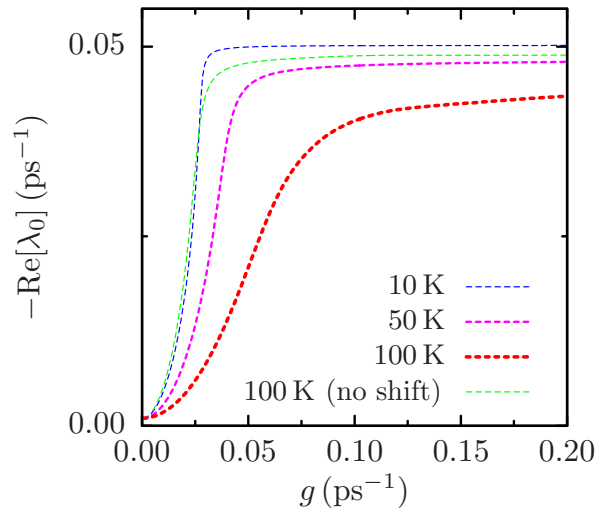

FIG. 4. (Color online) Real eigenvalue $\lambda_{0}$ of the matrix $M$ in Eq. (1) calculated for the same parameters and conditions as $\lambda_{ \pm}$ shown in Fig. 3.

$g$ dependence of $\gamma$ that again arises from the resonant nature of the carrier-phonon coupling $[14,38,39]$.

Let us turn now to the $\lambda_{0}$ mode and the corresponding results displayed in Fig. 4. It is clearly seen that the damping of the $\lambda_{0}$ mode increases with rising $g$ but it decreases with rising temperature. We stress that this temperature dependence is only introduced by the competition between cavity losses and phonon-induced pure dephasing: cavity losses alone would not lead to a $T$ dependence in our model, as $T$ enters only in the phonon-induced parts. Pure dephasing alone would also not lead to a $T$ dependence, as Eq. (3) predicts no damping of the $\lambda_{0}$ mode at all.

So far, our discussion has been restricted to the dependences of the eigenvalues of the matrix $M$. The eigenvalues define the positions and the widths of the spectral components into which the elements of the density matrix can be decomposed but do not reveal the relative weights that enter a specific observable. As an example of how the above-discussed dependences of the eigenvalues translate into properties of a measurable quantity, we shall discuss the real part of the Fourier transform of the dot exciton occupation $\rho_{11}(\omega)$ obtained by solving Eq. (1) for the system initially prepared in state $|2\rangle$. Experimentally, the dot occupation in the time regime is accessible in photocurrent, pump-probe, or transient-reflectivity measurements [40-44] from which $\rho_{11}(\omega)$ can be obtained by performing the Fourier transformation.

Figure 5 shows the corresponding spectra for different values of $g$ chosen above the bifurcation point where each spectrum exhibits a three-peak structure. It is clearly seen that the width of the side peaks increases with rising $g$ for small or moderate $g$, while for very high $g$ they decrease. This is accompanied by similarly nonmonotonic changes of the peak heights, which first decrease before increasing again at large $g$. As this nonmonotonic behavior follows from that of $\gamma(g, T)$, it is also observed in a system with negligible photon losses [14]. For comparison, results for the latter case are also displayed in Fig. 5 by dashed lines. The competition with cavity losses overall reduces the heights and increases the widths of the peaks but does not destroy the phonon-induced nonmonotonic dependence on $g$.

The temperature dependence resulting from the combination of cavity losses and phonon-induced pure dephasing also 


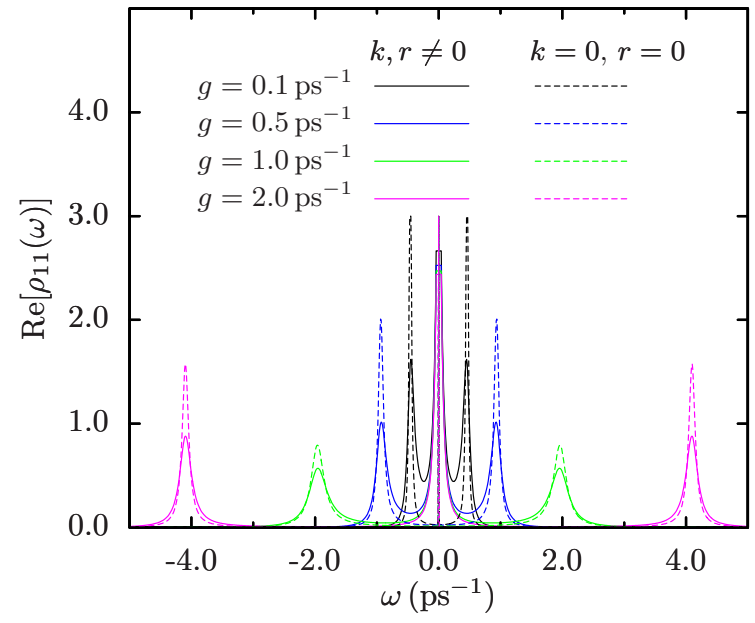

FIG. 5. (Color online) Solid lines: spectra $\operatorname{Re}\left[\rho_{11}(\omega)\right]$ calculated for different values of the dot-cavity coupling $g$ at $T=10 \mathrm{~K}$ and $\kappa=0.1, r=0.001 \mathrm{ps}^{-1}$. Dashed lines: $\operatorname{Re}\left[\rho_{11}(\omega)\right]$ calculated for $\kappa=$ $r=0$ with otherwise identical parameters. The heights of all central peaks as well as of the side peaks for $g=0.1 \mathrm{ps}^{-1}$ and $\kappa=r=0$ (dashed black line) are clipped to improve the visibility of the other side peaks.

affects the width of the peak centered at $\omega=0$ in the spectra $\operatorname{Re}\left[\rho_{11}(\omega)\right]$. Figure 6(a) plots the corresponding full width at half maximum (FWHM) that is extracted for different $T$ as a function of $g$ from calculations of $\operatorname{Re}\left[\rho_{11}(\omega)\right]$. It should be noted that the $\lambda_{ \pm}$modes also contribute to the width of that peak as long as the corresponding frequencies are not well separated from $\omega=0$ [see Figs. 6(b) and 6(c)]. This

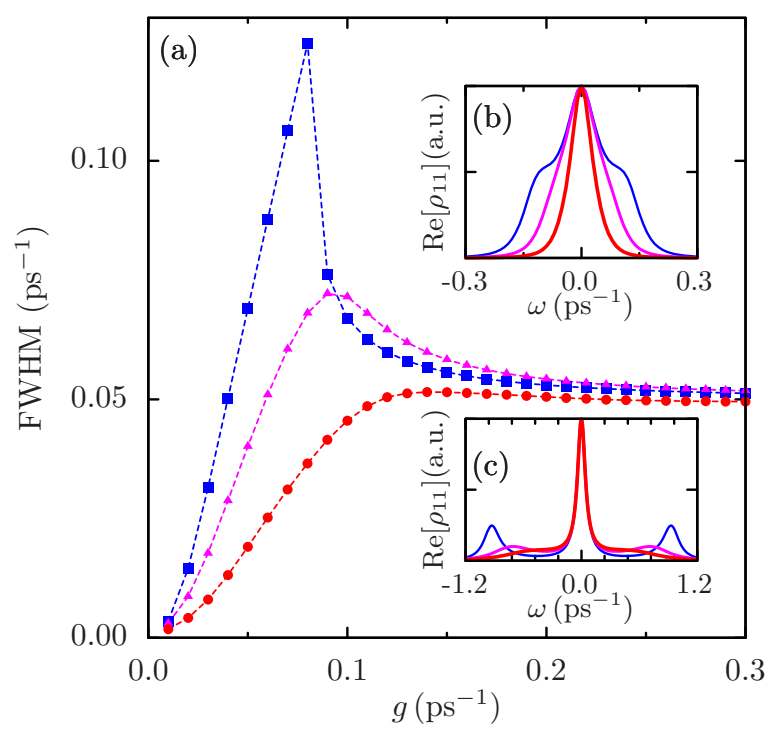

FIG. 6. (Color online) (a) Full width at half maximum (FWHM) of the central peak in the spectra $\operatorname{Re}\left[\rho_{11}(\omega)\right]$ as a function of the dot-cavity coupling $g$ calculated at $T=10 \mathrm{~K}$ (blue dashed line with squares), $T=50 \mathrm{~K}$ (purple dashed line with triangles), and $T=$ $100 \mathrm{~K}$ (red dashed line with circles). Triplet spectra at $T=10$ (thin blue line), $T=50 \mathrm{~K}$ (purple line), and $T=100 \mathrm{~K}$ (thick red line) calculated for (b) $g=0.075 \mathrm{ps}^{-1}$ and (c) $g=0.15 \mathrm{ps}^{-1}$. In all panels, $\kappa=0.1 \mathrm{ps}^{-1}$ and $r=0.001 \mathrm{ps}^{-1}$. applies in particular to the weak-coupling limit, where the $\lambda_{ \pm}$ contributions are centered at $\omega=0$ as well. For $T=100 \mathrm{~K}$, the heights of the peaks associated with the $\lambda_{ \pm}$modes are generally low, and thus, the FWHM in Fig. 6(a) reflects only the monotonically rising contribution of the $\lambda_{0}$ mode. For lower temperatures, a maximum appears because at low $g$, the $\lambda_{ \pm}$contributions become more important and strongly enhance the FWHM as their damping typically increases with rising temperature (see Fig. 3). This influence decreases with increasing $g$, when the side peaks move to higher $\omega$ and eventually become clearly separated.

Due to the competition between the $\lambda_{0}$ and the $\lambda_{ \pm}$ contributions, the $T$ dependence of the FWHM becomes nonmonotonic at intermediate $g$, while at high $g$, where the $\lambda_{ \pm}$ modes are no longer contributing, the low- $g$ trend, reflecting the dependence of the $\lambda_{0}$ mode, is restored; that is, we find a decrease of the width with rising temperature.

The temperature dependence of the $\lambda_{0}$ mode which has been shown to govern the observable width of the central peak in the spectra $\operatorname{Re}\left[\rho_{11}(\omega)\right]$ can be understood intuitively at least in the limit of small $g$. To this end, we recall that $\lambda_{0}$ is the eigenvalue closest to zero. In the limit $g \rightarrow 0$ this mode describes the incoherent decay either of $\rho_{11}$ (for $r<\kappa$ ) or of $\rho_{22}$ (for $r>\kappa$ ); in our case the eigenvector of the $\lambda_{0}$ mode has, at $g=0$, a nonzero component only for $\rho_{11}$. Increasing $g$ to a finite value introduces a transfer between the occupations $\rho_{11}$ and $\rho_{22}$ and thus couples any excitation in the subspace spanned by $|1\rangle$ and $|2\rangle$ to the faster of the two loss channels provided by $\kappa$ and $r$. Consequently, the slowest decay of an excitation in this subspace (this is the decay described by $\lambda_{0}$ ) speeds up due to the coupling to the faster decay channel. The temperature dependence of $\lambda_{0}$ can now be traced back to the $T$ dependences of $\tilde{g}$ and $\gamma$ displayed in Fig. 2. For low $g$ the renormalized light-matter coupling $\tilde{g}$ is strongly reduced, and thus, the coupling between $\rho_{11}$ and $\rho_{22}$, which speeds up the decay of the slowest decaying mode, is suppressed for higher temperatures. The role of the pure dephasing rate $\gamma$ can be appreciated by noting that according to Eq. (1), the occupation transfer between $\rho_{11}$ and $\rho_{22}$ is mediated by the coherences $V=\rho_{12}-\rho_{21}$. The increase of $\gamma$ with temperature seen in Fig. 2 leads to a stronger damping of the coherences when $T$ is increased and thus to a reduction of the excitation transfer. Thus, the reduction of $\tilde{g}$ and the increase of $\gamma$ with rising temperature both suppress the transfer between $\rho_{11}$ and $\rho_{22}$, which is the physical origin of the temperature dependence of $\lambda_{0}$.

Finally, we would like to address briefly a practical issue. In order to deal with a well-defined situation that is most favorable for the observation of vacuum Rabi oscillations that indicate the strong-coupling regime, we have assumed the cavity is strictly in resonance with the dot transition. However, it is well known that the dot transition energy shifts with temperature $[32,45]$. If this shift is not compensated, a change in temperature would detune the dot from the cavity, making it practically impossible to observe a crossover between the weak- and strong-coupling regimes. Therefore, in order to see the temperature dependences caused by the interplay of phonon-induced pure dephasing and cavity losses, the problem arises of how to maintain the resonance condition while changing the temperature. There are several possible 
solutions to this problem. For example, experiments indicate that dot transitions can be tuned in and out of resonance with a cavity by applying suitable static electric or magnetic fields [45-47]. However, when such methods are used to readjust the transition energy, a quantitative description may require us to account for the changes of the dot-phonon couplings induced by the applied fields. This can, in principle, be avoided, e.g., by dielectric switching of the frequency of the cavity to restore the resonance conditions [48]. The feasibility of such switching strategies has been experimentally demonstrated for cavities without dots $[49,50]$.

\section{CONCLUSIONS}

In conclusion we have shown that the interplay between cavity losses and the $g$-dependent pure dephasing and the phonon-induced renormalization of the bare light-matter coupling strength $g$ strongly affect the transition from the weak- to the strong-coupling regime, e.g., reflected in
$T$-dependent shifts of the bifurcation point. In the weakcoupling limit, we predict that the width of the peak at $\omega=0$ in the Fourier representation of the dot occupation decreases for higher temperatures. Our analysis reveals that the temperature dependence of this line results from the combined action of cavity losses and phonon-induced pure dephasing; either mechanism alone would lead to a temperature-independent width.

\section{ACKNOWLEDGMENTS}

We acknowledge fruitful discussions with A. Nazir which helped us to more clearly formulate the relation between our phenomenological approach and the microscopic theory. M.D.C. further acknowledges Alexander von Humboldt and BELSPO grants for support. Financial support from the Deutsche Forschungsgemeinschaft (Grant No. AX 17/7-1) is also gratefully acknowledged.
[1] D. Press, S. Götzinger, S. Reitzenstein, C. Hofmann, A. Löffler, M. Kamp, A. Forchel, and Y. Yamamoto, Phys. Rev. Lett. 98, 117402 (2007).

[2] A. Dousse, J. Suffczyński, A. Beveratos, O. Krebs, A. Lemaître, I. Sagnes, J. Bloch, P. Voisin, and P. Senellart, Nature (London) 466, 217 (2010).

[3] M. Müller, S. Bounouar, K. D. Jöns, M. Glässl, and P. Michler, Nat. Photon. 8, 224 (2014).

[4] D. Bouwmeester, A. K. Ekert, and A. Zeilinger, The Physics of Quantum Information (Springer, Berlin, 2000).

[5] S. Haroche and J. Raimond, Exploring the Quantum (Oxford University Press, Oxford, 2006).

[6] C. Boeckler, S. Reitzenstein, C. Kistner, R. Debusmann, A. Loeffler, T. Kida, S. Hfling, A. Forchel, L. Grenouillet, J. Claudon et al., Appl. Phys. Lett. 92, 091107 (2008).

[7] S. Ates, S. M. Ulrich, A. Ulhaq, S. Reitzenstein, A. Löffler, S. Höfling, A. Forchel, and P. Michler, Nat. Photon. 3, 724 (2009).

[8] P. Kaer, T. R. Nielsen, P. Lodahl, A. P. Jauho, and J. Mørk, Phys. Rev. Lett. 104, 157401 (2010).

[9] S. Hughes, P. Yao, F. Milde, A. Knorr, D. Dalacu, K. Mnaymneh, V. Sazonova, P. J. Poole, G. C. Aers, J. Lapointe et al., Phys. Rev. B 83, 165313 (2011).

[10] S. M. Ulrich, S. Ates, S. Reitzenstein, A. Löffler, A. Forchel, and P. Michler, Phys. Rev. Lett. 106, 247402 (2011).

[11] C. Roy and S. Hughes, Phys. Rev. Lett. 106, 247403 (2011).

[12] P. Kaer, P. Lodahl, A.-P. Jauho, and J. Mørk, Phys. Rev. B 87, 081308 (2013).

[13] Z. Harsij, M. Bagheri Harouni, R. Roknizadeh, and M. H. Naderi, Phys. Rev. A 86, 063803 (2012).

[14] M. Glässl, L. Sörgel, A. Vagov, M. D. Croitoru, T. Kuhn, and V. M. Axt, Phys. Rev. B 86, 035319 (2012).

[15] A. Carmele, M. Richter, W. W. Chow, and A. Knorr, Phys. Rev. Lett. 104, 156801 (2010).

[16] A. Carmele, J. Kabuss, and W. W. Chow, Phys. Rev. B 87, 041305 (2013).
[17] D. P. S. McCutcheon and A. Nazir, Phys. Rev. Lett. 110, 217401 (2013).

[18] B. Krummheuer, V. M. Axt, and T. Kuhn, Phys. Rev. B 65, 195313 (2002).

[19] A. Krügel, A. Vagov, V. M. Axt, and T. Kuhn, Phys. Rev. B 76, 195302 (2007).

[20] J. Förstner, C. Weber, J. Danckwerts, and A. Knorr, Phys. Rev. Lett. 91, 127401 (2003).

[21] A. Krügel, V. M. Axt, T. Kuhn, P. Machnikowski, and A. Vagov, Appl. Phys. B 81, 897 (2005).

[22] F. Milde, A. Knorr, and S. Hughes, Phys. Rev. B 78, 035330 (2008).

[23] A. J. Ramsay, T. M. Godden, S. J. Boyle, E. M. Gauger, A. Nazir, B. W. Lovett, A. M. Fox, and M. S. Skolnick, Phys. Rev. Lett. 105, 177402 (2010).

[24] A. Auffèves, D. Gerace, J.-M. Gérard, M. F. Santos, L. C. Andreani, and J.-P. Poizat, Phys. Rev. B 81, 245419 (2010).

[25] C. C. Gerry and P. L. Knight, Introductory Quantum Optics (Cambridge University Press, Cambridge, 2005).

[26] G. D. Mahan, Many-Particle Physics, 2nd ed. (Plenum, New York, 1990).

[27] S. Mukamel, Principles of Nonlinear Optical Spectroscopy (Oxford University Press, New York, Oxford, 1995).

[28] H. P. Breuer and F. Petruccione, The Theory of Open Quantum Systems (Oxford University Press, Oxford, 2002).

[29] P. Kaer, T. R. Nielsen, P. Lodahl, A.-P. Jauho, and J. Mørk, Phys. Rev. B 86, 085302 (2012).

[30] L. C. Andreani, G. Panzarini, and J. M. Gerard, Phys. Rev. B 60, 13276 (1999).

[31] S. Rudin and T. L. Reinecke, Phys. Rev. B 59, 10227 (1999).

[32] J. P. Reithmaier, G. Sek, A. Löffler, C. Hofmann, S. Kuhn, S. Reitzenstein, L. V. Keldysh, V. D. Kulakovskii, T. L. Reinecke, and A. Forchel, Nature (London) 432, 197 (2004).

[33] K. Hennessy, A. Badolato, M. Winger, D. Gerace, M. Atatüre, S. Gulde, S. Fält, E. L. Hu, and A. Imamoğlu, Nature (London) 445, 896 (2007). 
[34] M. Thorwart, J. Eckel, and E. R. Mucciolo, Phys. Rev. B 72, 235320 (2005).

[35] M. Glässl, A. Vagov, S. Lüker, D. E. Reiter, M. D. Croitoru, P. Machnikowski, V. M. Axt, and T. Kuhn, Phys. Rev. B 84, 195311 (2011).

[36] A. Vagov, M. D. Croitoru, M. Glässl, V. M. Axt, and T. Kuhn, Phys. Rev. B 83, 094303 (2011).

[37] The carrier-phonon coupling constants are taken from Ref. [18].

[38] P. Machnikowski and L. Jacak, Phys. Rev. B 69, 193302 (2004).

[39] A. Vagov, M. D. Croitoru, V. M. Axt, T. Kuhn, and F. M. Peeters, Phys. Rev. Lett. 98, 227403 (2007).

[40] M. Wesseli, C. Ruppert, S. Trumm, H. J. Krenner, J. J. Finley, and M. Betz, Appl. Phys. Lett. 88, 203110 (2006).

[41] Y. Mitsumori, Y. Miyahara, K. Uedaira, H. Kosaka, S. Shimomura, S. Hiyamizu, and K. Edamatsu, Jpn. J. Appl. Phys. 50, 095004 (2011).

[42] C. Wolpert, C. Dicken, P. Atkinson, L. Wang, A. Rastelli, O. G. Schmidt, H. Giessen, and M. Lippitz, Nano Lett. 12, 453 (2012).
[43] C. Wolpert, C. Dicken, L. Wang, P. Atkinson, A. Rastelli, O. G. Schmidt, H. Giessen, and M. Lippitz, Phys. Status Solidi B 249, 721 (2012).

[44] J. H. Quilter, R. J. Coles, A. J. Ramsay, A. M. Fox, and M. S. Skolnick, Appl. Phys. Lett. 102, 181108 (2013).

[45] S. Reitzenstein, S. Münch, P. Franeck, A. Rahimi-Iman, A. Löffler, S. Höfling, L. Worschech, and A. Forchel, Phys. Rev. Lett. 103, 127401 (2009).

[46] C. Kistner, T. Heindel, C. Schneider, A. Rahimi-Iman, S. Reitzenstein, S. Höfling, and A. Forchel, Opt. Express 16, 15006 (2008).

[47] H. Kim, D. Sridharan, T. C. Shen, G. S. Solomon, and E. Waks, Opt. Express 19, 2589 (2011).

[48] H. Thyrrestrup, A. Hartsuiker, J.-M. Gérard, and W. Vos, Opt. Express 21, 23130 (2013).

[49] P. J. Harding, T. Euser, Y. Nowicki-Bringuier, J.-M. Gérard, and W. Vos, Appl. Phys. Lett. 91, 111103 (2007).

[50] T. G. Euser, A. J. Molenaar, J. G. Fleming, B. Gralak, A. Polman, and W. L. Vos, Phys. Rev. B 77, 115214 (2008). 